\title{
Introducción de estrategias para la mejora de la retroalimentación formativa en la asignatura de Laboratorio de Desarrollo Industrial del Grado en Ingeniería Química
}

\author{
Carmen B. Molina, Pablo Navarro, Gema Pliego, Jesús Lemus, Francisco Heras, \\ Asunción Quintanilla
}

Departamento de Ingeniería Química. Facultad de Ciencias. Universidad Autónoma de Madrid. carmenbelen.molina@uam.es; pablo.navarro@uam.es; gema.pliego@uam.es; jesus.lemus@uam.es; fran.heras@uam.es; asun.quintanilla@uam.es

\begin{abstract}
This work has been carried out for the Industrial Development Laboratory subject (4th course, Chemical Engineering Degree, Autonoma University of Madrid). The student is in his/her last academic year of the bachelor and he/she should possess the maturity and knowledge necessary to face the subject with sufficient autonomy and initiative. However, in the last years, it has been detected that the project-based teaching metodology does not ensure the skill acquisition by all of the members of the same work-team because not all students assume the same degree of responsibility in their own learning.

Therefore, the objective of this work is to enrich the formative feedback of the students enrolled in this experimental subject. For this, efficient working groups have been created, new teaching methodologies have been introduced and a project management tool has been implemented (Trello).

The impact of the introduction of these strategies has been evaluated based on both the results of surveys, aimed at knowing about the students' perception towards the new methodologies, and the analysis of learning outcome, compared to previous courses. The activities that have contributed the most to improve the teacher feedback have been the face-to-face tutoring, while teamwork and laboratory practices, the peer feedback.
\end{abstract}

Keywords: Active methodology learning, Feedback, Self-evaluation, Industrial Development Laboratory

\section{Resumen}

Este trabajo se enmarca en la asignatura Laboratorio de Desarrollo Industrial ( $4^{\circ}$ Grado en Ingeniería Química, Universidad Autónoma de Madrid). En los últimos años, se han detectado diversos aspectos en los que el enfoque del aprendizaje basado en proyectos no asegura la adquisición de competencias por todos los miembros de un mismo equipo de trabajo, debido a que no todos los estudiantes asumen el mismo grado de responsabilidad en su aprendizaje.

Por ello, el objetivo del presente trabajo es enriquecer la retroalimentación formativa de los estudiantes de esta asigntura experimental. Para ello se han creado grupos de trabajo eficaces, se han introducido nuevas metodologías docentes y se ha implementado una herramienta de gestión de proyectos (Trello).

El impacto de la introduccion de estas estrategias para la mejora de la retroalimentaicon formativa se ha evaluado en base al resultado de encuestas orientadas a conocer la percepción de los estudiantes sobre

(cc) BY-NC-ND 2020, Universitat Politècnica de València

Congreso In-Red (2020) 
las nuevas metodologías introducidas y al análisis de los resultados de aprendizaje en comparación con cursos anteriores. Las actividades que más han contribuido a fomentar la retroalimentación del profesorado han sido las tutorías presenciales, mientras que el trabajo en equipo y las prácticas de laboratorio, la retroalimentación de los compañeros.

Palabras clave: Metodologías de aprendizaje activos, Retroalimentación, Autoevaluación, Laboratorio de Desarrollo Industrial, Grado en Ingeniería Química.

\section{Introducción}

La asignatura Laboratorio de Desarrollo Industrial (LDI) es una asignatura obligatoria de 6 créditos ECTS, que se imparte durante el primer cuatrimestre del $4^{\circ}$ curso del Grado en Ingeniería Química de la Universidad Autónoma de Madrid (UAM). Pertenece a la materia Laboratorio Integrado de Ingeniería Química y es de carácter práctico junto a otras dos asignaturas del Grado: Experimentación en Ingeniería $\left(2^{\circ}\right.$ curso) y Experimentación en Ingeniería Química ( $3^{\mathrm{er}}$ curso).

La asignatura LDI está planificada de acuerdo a la metodología de aprendizaje colaborativo basado en proyectos (Basilotta, 2013; Martí, 2010). Los estudiantes se dividen en grupos, de 3-4 alumnos, para resolver un problema industrial que implica el diseño básico de un equipo de proceso, como una columna de separación o un reactor químico, para satisfacer unas especificaciones de proceso y producto. Para ello disponen de una planta piloto del equipo industrial en cuestión, en la que deben planificar y realizar los experimentos necesarios para estudiar los fenómenos que tienen lugar en el interior del equipo a diseñar (fluidodinámica, transferencia de materia y reacción química) y obtener así la información necesaria para modelar la operación o reacción quimica. Conocido el modelo y en base a las balances de materia y energía, podrán simular el comportamiento del equipo en planta piloto en las condiciones de operación empleadas, así como en las condiciones indicadas en el caso real supuesto. De esta manera podrán abordar el escalado, la estimación de costes, la propuesta de mejoras y la selección de la mejor alternativa.

Durante su periodo de aprendizaje, los estudiantes contarán con la tutorización de un profesor que les guiará durante el todo el desarrollo de u proyecto. El trabajo que deben realizar en equipo es intenso ya que entre otras tareas se espera que los grupos: se documenten sobre el proceso a estudiar, planifiquen experimentos, realicen cálculos, realicen un escalado y diseño de la instalación a escala industrial, calculen los costes y diseñen el sistema de control del proceso completo.

Los proyectos son asignados aleatoriamente desde el principio de curso a los distintos grupos. La clase se divide en dos turnos de trabajo, y en cada turno se reparten los mismos proyectos (un total de 7), de manera que dos grupos van a desarrollar el mismo proyecto, pero en turnos diferentes y, por tanto, con tutores diferentes. Las actividades presenciales son seminarios sobre los conocimientos teóricos necesarios para abordar el desarrollo del proyecto, 7 días de experimentación en la planta piloto y 4 tutorías de seguimiento programadas. Estas tutorías son reuniones temáticas de discusión en la que se lleva a cabo una evaluación por pares de docentes del trabajo que van realizando los alumnos. Los dos grupos que tienen asignados el mismo proyecto exponen su trabajo (conforme a unos objetivos especificados antes de la tutoría) y son evaluadas por los dos tutores. La evaluación de la asignatura se realiza atendiendo a las calificaciones de estas 4 tutorías de seguimiento, al informe final y a la defensa oral del proyecto al final del cuatrimestre, al trabajo del alumno en el laboratorio y un examen final escrito individual que permite comprobar los conocimientos adquiridos por el estudiante tras la realización de la asignatura. 
Dado el aprendizaje autónomo que se espera de los estudiantes para la adquisición de las nuevas competencias de esta asignatura, parece fundamental garantizar que los grupos de trabajo sean eficientes y puedan abordar el trabajo desde el inicio de manera fructífera (París, 2016; Lerís, 2017). Por otro lado, es esencial disponer de herramientas que favorezcan la retroalimentación formativa de los estudiantes para que ayuden a mantener su compromiso de aprendizaje y grado de responsabilidad en el grupo, así como para trabajar de manera continuada a lo largo del cuatrimestre.

\section{Objetivos}

El presente proyecto de Innovación Docente tiene por objetivo enriquecer la retroalimentación formativa de los estudiantes de la asignatura Laboratorio de Desarrollo Industrial, atendiendo a dos enfoques diferentes, retroalimentación de sus propios compañeros de trabajo y retroalimentación de su tutor. Para ello se van a crear grupos de trabajo eficaces, en base a los conocimientos iniciales de los estudiantes, su actitud y aptitud para trabajar en equipo y su relación con los compañeros de clase (sociogramas). Además, se van a introducir nuevas metodologías docentes orientadas a: fomentar la autonomía en el aprendizaje de todos los miembros del grupo, fomentar una adquisición progresiva de competencias, motivar al trabajo continuado en equipo y ayudarles a tomar conciencia de sus propias capacidades para que valoren y aprecien las capacidades del resto de miembros del grupo. Por último, se va a implementar una herramienta de gestión de proyectos que ayuden a la organización del trabajo, al desarrollo progresivo del trabajo a lo largo de todo el cuatrimestre y a la tutorización no presencial del mismo por parte de los docentes.

La acción propuesta se enmarca dentro de las áreas de incorporación de metodologías activas, evaluación inicial de conocimientos y competencias, desarrollo de instrumentos de evaluación formativa o autoevaluación e implantación de estrategias de aprendizaje basadas en las tecnologías actuales. La implementación y desarrollo de las nuevas metodologías propuestas ayudará al estudiante a obtener una mayor autonomía en el aprendizaje, motivándoles para realizar sus tareas, ayudándoles a tomar conciencia de sus propias capacidades y obtener una mayor igualdad entre los diferentes grupos de trabajo.

\section{Desarrollo de la Innovación}

Las actuaciones propuestas en este trabajo se han aplicado a la asignatura obligatoria de carácter experimental Laboratorio de Desarrollo Industrial (LDI). El número de alumnos matriculados en los pasados cursos, desde el 16/17 hasta el actual -19/20-, ha sido de 41, 55, 60 y 52, respectivamente.

El desarrollo del proyecto se ha llevado a cabo actuando en diversas etapas del desarrollo de la asignatura. Las nuevas metodologías docentes desarrolladas han sido las siguientes:

\subsection{Formación de grupos de trabajo eficaces}

Los grupos de trabajo que formaban los propios estudiantes en cursos anteriores, por afinidades personales, resultaban poco equilibrados, ya que solían estar formados por estudiantes con buen expediente, y otros con estudiantes con expedientes más mediocres, de manera que era difícil que aprendieran unos de otros y aprovecharan la esencia del trabajo colaborativo.

Con objeto de formar grupos de trabajo más eficaces, durante los primeros días del curso se elaboró un cuestionario inicial de autoevaluación de los alumnos que permitiera establecer un perfil de capacidades y competencias. Dicho cuestionario se cumplimentó en Google Formulario el primer día de clase. De esta forma se pudieron configurar grupos de trabajo mediante criterios de roles de equipo para compensar las competencias que cada estudiante asume en el mismo, así como su nivel académico. Ésta ha sido una gran 
novedad en el transcurso de la asignatura en este curso ya que, hasta ahora, los grupos los formaban los propios estudiantes, por afinidades personales, dando lugar a grupos que obtenían muy buenas calificaciones y otros con más bajas ya que la tendencia de los estudiantes es, generalmente, a agruparse por nivel académico.

En este primer cuestionario inicial para la formación de grupos se les preguntó sobre diferentes aspectos como la nota media global de los anteriores cursos, nota media de asignaturas cursadas en anteriores cursos y directamente implicadas en el desarrollo de esta asignatura de cuarto curso, preferencia a trabajar en equipo o no, a realizar un trabajo experimental o uno de diseño, si se consideran organizados a la hora de trabajar, si ante una dificultad prefieren buscar asesoramiento o tomar la iniciativa y si en una situación de conflicto tratan de encontrar soluciones pudiendo anteponer los intereses del grupo a los suyos propios. Asimismo, se les pidió que valorasen sus capacidades en diferentes aspectos tratados en la asignatura como expresión oral, escrita, diseño de unidades de separación o reactores y realización de tareas experimentales en laboratorio entre otros.

Además, se les pidió que rellenaran unos cuestionarios de afinidad personal para trabajar en equipo con el resto de estudiantes de la clase mediante la aplicación informática Sometics, cuyos resultados también se tuvieron en cuenta a la hora de formar los grupos de trabajo.

\subsection{Cuestionarios de autoevaluación previos a las tutorías de seguimiento.}

En esta asignatura se realizan diferentes actividades formativas entre las que se encuentran 4 tutorías de seguimiento del trabajo en las que, por grupos, los estudiantes exponen ante dos profesores de la asignatura diferentes partes del trabajo desarrollado en la asignatura y, posteriormente, se hace un turno de preguntas por parte de los profesores a los estudiantes.

Unos días antes a la realización de cada una de las 4 tutorías de seguimiento los estudiantes dispusieron de un breve cuestionario vía Moodle con 5 preguntas tipo test con los contenidos clave de la siguiente tutoría de modo que ellos mismos puedan autoevaluarse (obtenía el resultado tres evaluarse). De este modo se medía el grado de preparación del estudiante ante las inminentes tutorías de seguimiento a la vez que el propio estudiante podía comprobar si estaba preparado o no para afrontarla, sirviendo de herramienta de autoevaluación.

\subsection{Cuestionarios autoevaluación y coevaluación entre grupos distintos que trabajen en el mismo proyecto.}

Durante las tutorías de seguimiento se reúnen en el mismo aula los dos grupos que realizan el mismo problema industrial, pero que abordan por separado y tutorizados por diferentes docentes. Cada grupo dispone de cierto tiempo de presentación seguido de un tiempo en el que los docentes realizan preguntas de evaluación. Al finalizar dichas tutorías los estudiantes rellenaron cuestionarios de coevaluación (mediante Formularios de Google), evaluando al otro grupo que había expuesto, y de autoevaluación en el que los estudiantes daban una calificación a su propia actuación. De este modo, los estudiantes asumen la responsabilidad de evaluar tanto a los compañeros de práctica como a sí mismos. Dichas notas fueron comparadas posteriormente con las dadas por los dos docentes de la asignatura que evalúan cada tutoría.

\subsection{Implementación de Trello como herramientas de gestión de proyectos.}

Se ha puesto a disposición de los estudiantes la posibilidad de comunicarse entre ellos y con el profesor tutor de su práctica a través del programa Trello, software de administración de proyectos con interfaz web y con cliente para iOS y Android. Esta herramienta es empleada frecuentemente por algunos docentes en 
otras asignaturas, habiéndose comprobado su accesibilidad para los estudiantes y permitiendo crear hojas de ruta adaptadas a cada grupo de trabajo, así como una tutorización y retroalimentación continua. Además, con esta herramienta se puede organizar la información que recopilan, así como bibliografía, planificación de experimentos, hojas de cálculo e informes que elaboran, teniendo acceso los tutores a todo ello.

Finalmente, y tras haber desarrollado todas las actividades formativas de las que consta esta asignatura, se facilitó un cuestionario a los estudiantes para comprobar su percepción sobre las nuevas metodologías introducidas en la misma. En dicho cuestionario se les preguntó sobre su nivel de implicación en la asignatura, cómo la realización de esta asignatura ha permitido mejorar sus capacidades en los mismos aspectos que en la encuesta inicial (para comprobar su evolución) y se les pidió que valoraran las diferentes actividades realizadas en la asignatura como la formación de los grupos de trabajo, empleo de la herramienta informática Trello, tutorías presenciales y presenciales evaluativas, prácticas de laboratorio, cuestiones previas a las tutorías, cuestionarios de co y autoevaluación y seminarios iniciales (seminarios teóricos impartidos antes de la experimentación en planta piloto). Asimismo, se les preguntó por su satisfacción global en relación a la asignatura y se les dio la posibilidad de añadir alguna recomendación constructiva.

\section{Resultados}

\subsection{Formación de los grupos de trabajo de estudiantes en base a un cuestionario inicial de nivel de aprendizaje, criterios de roles de equipo y afinidad personal.}

Con objeto de llevar a cabo la formación de grupos de trabajo eficaces se pasó a los estudiantes un cuestionario inicial que recogía varios aspectos: calificación media de asignaturas realizadas en cursos anteriores muy relacionadas con la base teórica de LDI y otras cuestiones sobre el rol que podrían desempeñar en el grupo de trabajo. Respecto a la calificación media de las asignaturas cursadas en anteriores cursos relacionadas con la base teórica LDI, dos asignaturas de $3^{\text {er }}$ curso sobre Ingeniería de las Reacciones Químicas y otra de $3^{\text {er }}$ curso sobre Operaciones de Separación, en ambos casos más de la mitad de los estudiantes tenía como calificación únicamente Aprobado e incluso alrededor del $10 \%$ de los estudiantes tenían suspensa alguna de estas asignaturas, esenciales para el buen desarrollo de la asignatura de LDI al tratar la base teórica necesaria para la misma (Figura 1).

Respecto a las preguntas del cuestionario que inciden sobre las preferencias de los estudiantes para adquirir distintos roles dentro del grupo de trabajo, a la mayoría de los estudiantes les gusta trabajar en equipo (casi un 90\%), prefieren trabajo experimental frente a los de diseño (más del 66\%), se consideran personas organizadas (casi un $80 \%$ ), prefieren buscar asesoramiento externo antes de tomar ellos la iniciativa (más del 60\%) y prefieren encontrar soluciones a los conflictos, anteponiendo los intereses del grupo al suyo propio (casi un $86 \%$ ). Por lo tanto, los roles para trabajar en el grupo son en su mayoría comunes a gran parte de los estudiantes.
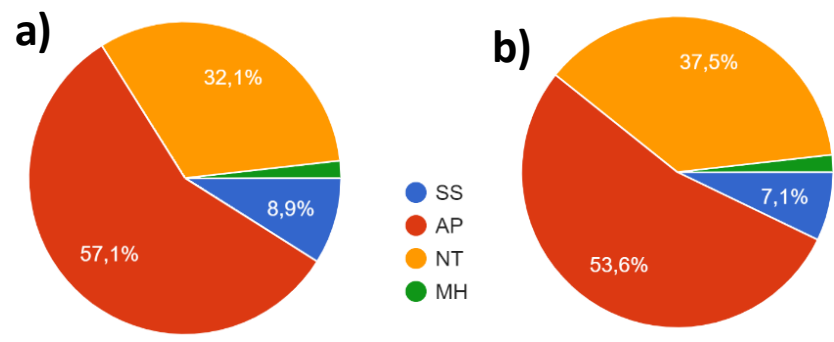

Figura 1. Calificaciones medias en asignaturas base de cursos anteriores: a) Ingeniería de las Reacciones Quimicas, b) Operaciones de Separación.

(cc) BY-NC-ND 2020, Universitat Politècnica de València

Congreso In-Red (2020) 
También se realizó a los estudiantes un cuestionario sociológico o de afinidades personales mediante la aplicación Sometics, en el que se les preguntó acerca de las personas que preferían tener o no en su grupo de trabajo desde un punto de vista social o de trabajo. El programa, a partir de esta información, formaba grupos de trabajo teniendo en cuenta, además, otros aspectos elegidos por los docentes, como "buen ambiente de trabajo", "inclusión de personas aisladas", "paridad en los grupos de trabajo" y "potenciación las relaciones de trabajo".

A partir de los cuestionarios realizados inicialmente a los estudiantes, su expediente académico y el cuestionario de afinidades personales se conformaron los grupos de trabajo. Sólo dos de los grupos formados de este modo mostraron problemas personales al inicio del trabajo, pero, posteriormente, dichos grupos fueron capaces de resolver sus diferencias y trabajaron de forma eficaz obteniendo, incluso en algunos casos, muy buenos resultados académicos. Sin embargo, esta forma de establecer los grupos de trabajo ha sido uno de los puntos peor valorados en la encuesta de percepción de la asignatura y valoración de actividades, ya que en muchos casos los compañeros de grupo seleccionados no son los que los estudiantes hubieran deseado para trabajar durante el desarrollo de la asignatura. Desde el punto de vista de los docentes, esta forma de conformar los grupos de trabajo ha sido positiva, habiendo logrado obtener grupos de trabajo más heterogéneos tanto a nivel académico como de capacidades personales $\mathrm{y}$, por tanto, que los grupos entre sí muestren mayor homogeneidad, dando lugar a muy buenos resultados académicos en la mayoría de los casos.

\subsection{Cuestionarios de autoevaluación previos a las tutorías de seguimiento.}

Se han obtenido calificaciones altas en estos cuestionarios, confirmando la buena preparación de los estudiantes antes de la realización de dichas tutorías de seguimiento. Además, dichas tutorías han servido para aclarar a los estudiantes los aspectos que los docentes consideran fundamentales. Además, los alumnos han mostrado mayor interés esos días, como veíamos con mayor frecuencia de uso de Trello.

\subsection{Cuestionarios autoevaluación y coevaluación entre grupos distintos que trabajen en el mismo proyecto.}

Comparando las calificaciones dadas por los estudiantes en ambos cuestionarios respecto a la de los profesores que evaluaron la tutoría (Figura 2) puede observarse cómo, en el caso de la autoevaluación, los estudiantes fueron más benevolentes y sus calificaciones se situaron por encima de las de los profesores, sobre todo en las tutorías I y II. En el caso de los cuestionarios de coevaluación, existe una mayor dispersión de datos, pero, en general, la calificación dada por los estudiantes fue superior a la dada por los profesores sobre todo en las primeras tutorías, I y II, mientras que, en las últimas tutorías, III y IV, los estudiantes fueron más conscientes de los fallos de sus compañeros y las calificaciones propuestas fueron incluso inferiores a la de los docentes, lo que demuestra un mayor grado de crítica hacia sus compañeros. De estos resultados se desprende que las tutorías I y II no son bien evaluadas por los estudiantes, probablemente porque al ser las primeras que realizan aún no conocen de forma adecuada las rúbricas que los docentes emplean para su evaluación, rúbricas que desde principio de curso están a disposición de los estudiantes en la plataforma Moodle de comunicación entre profesores y estudiantes. Se identifica como clave, por tanto, hacer hincapié en los próximos cursos por parte de los docentes para que los estudiantes no solo conozcan sino además comprendan y se familiaricen con dichas rúbricas. 

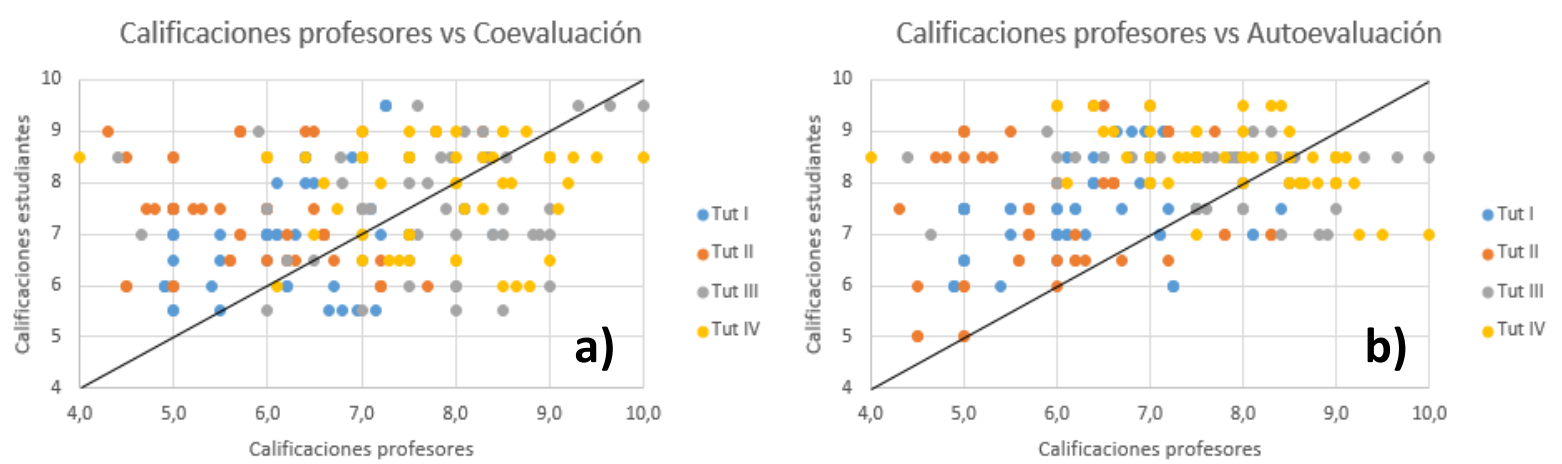

Figura 2. Comparación de las calificaciones de los cuestionarios de co y autoevaluación respecto a las calificaciones dadas por los profesores de la asignatura.

\subsection{Implementación de herramientas de gestión de proyectos, Trello.}

El grado de implementación de esta herramienta informática ha dependido enormemente del uso que de ella haya realizado el tutor. El empleo de esta herramienta ha ayudado a los estudiantes en la medida que el profesor la haya empleado para comunicarse con ellos y revisarles los cálculos e informes realizados, ya que los alumnos no mostraron iniciativa por su uso. En cualquier caso, las tutorías presenciales que han solicitado los estudiantes han disminuido notablemente durante el presente curso. En otros casos los grupos de trabajo se han comunicado con su tutor de la manera tradicional, prefiriendo concertar tutorías presenciales con los tutores para discutir los resultados y dudas en persona con el docente, aflorando también el intercambio de correos electrónicos como segunda vía más frecuentada por los alumnos.

\subsection{Cuestionario final de la asignatura para evaluar la percepción de los estudiantes.}

Terminada la asignatura se pasó un cuestionario final a los estudiantes que fue contestado de forma voluntaria por el $65 \%$ de los mismos, un número importante dado que no existía ningún mecanismo que motivara su realización. En dicho cuestionario se contemplaban diferentes aspectos de la asignatura.

En primer lugar, se les preguntó por su nivel de implicación en la asignatura considerando la mayor parte de los estudiantes que su implicación ha sido alta $(71 \%$ de los encuestados lo valoraron con 4 en una escala de 4$)$ o bastante alta ( $26 \%$, valoración de 3 en la escala de 4$)$.

Seguidamente se les preguntó sobre cómo se consideran a sí mismos de preparados en diferentes aspectos que se tratan en la asignatura. Esta misma pregunta se les hizo también en la encuesta inicial con objeto de comparar los resultados antes de haber cursado esta asignatura y después. En la Figura 3 se muestran los resultados de ambas encuestas. 


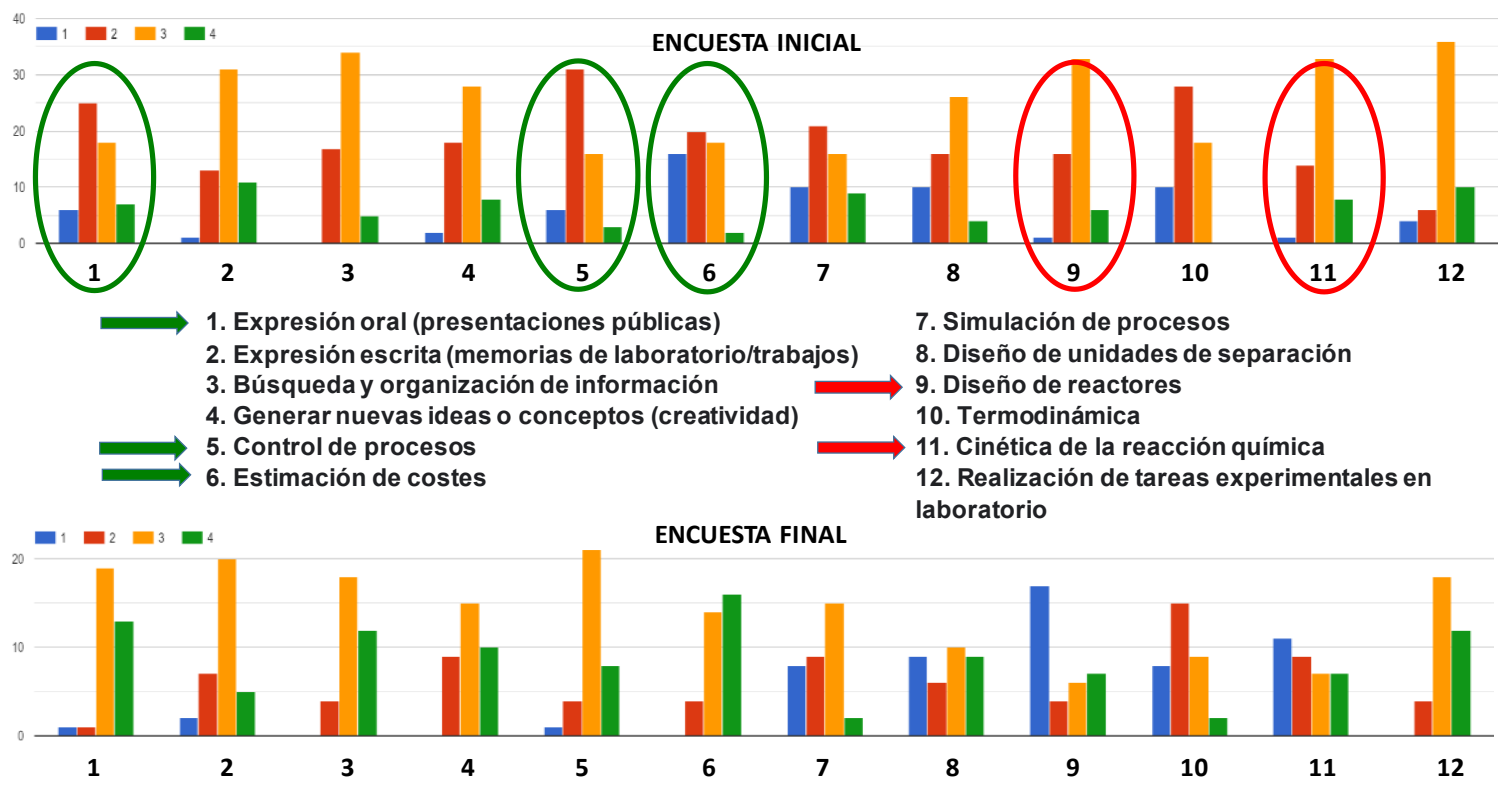

Figura 3. Evaluación de capacidades en diferentes actividades de la asignatura (calificaciones: 1 bajo, 4 alto)

Los aspectos en los que los estudiantes consideran que han mejorado tras cursar la asignatura son, principalmente, la expresión oral ya que han tenido que realizar 4 tutorías de seguimiento en las que la primera parte es una exposición oral del trabajo realizado en parte del proyecto y una defensa oral final resumiendo todo su trabajo, por lo que es una parte importante de la asignatura y los estudiantes así lo consideran. Asimismo, los estudiantes consideran que han mejorado, principalmente, en los temas de control de procesos y estimación de costes, aspectos muy trabajados a lo largo de la asignatura.

Respecto a la pregunta realizada en la encuesta final donde los estudiantes valoraron las diferentes actividades realizadas en la asignatura (Figura 4) son destacables algunas de las respuestas dadas por los estudiantes:

- Respecto a la formación de grupos de trabajo, la mayor parte de los estudiantes han contestado que ha mejorado su aptitud para el trabajo en equipo por lo que la visión de parte de los estudiantes no es tan negativa como la expresada por otros de sus compañeros.

- El empleo del programa Trello para mejorar la comunicación entre estudiantes y tutores es considerada por la mayoría de los estudiantes como que no ha sido útil en el desarrollo de la asignatura. Ello depende, en gran medida, del uso que el tutor haya hecho de esta herramienta para comunicarse con sus tutelados.

- Tanto las tutorías presenciales, únicamente con el tutor de la práctica, como las tutorías evaluativas en presencia de los dos profesores de la práctica, son valoradas positivamente y en su mayor parte los estudiantes consideran que han contribuido a mejorar su aprendizaje y a obtener una mayor retroalimentación por parte del profesor.

- Asimismo, tanto las prácticas de laboratorio como la introducción de los nuevos cuestionarios previos a las tutorías han sido considerados favorablemente por los estudiantes, contribuyendo a mejorar su aprendizaje y permitiéndoles ganar confianza en sus capacidades. 


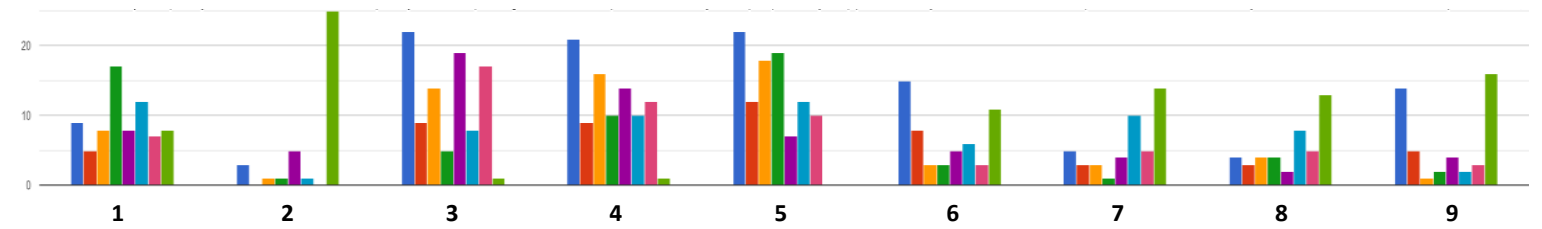

1. Formación de equipos de trabajo

2. Gestión de los proyectos en Trello

3. Tutorías presenciales

4. Tutorías presenciales evaluativas

5. Prácticas de laboratorio

6. Cuestionarios de autoevaluación Moodle previos a las tutorías

7. Cuestionarios (rúbricas) de coevaluación de las tutorías

8. Cuestionarios (rúbricas) de autoevaluación de las tutorías

9. Seminarios iniciales (clases magistrales)

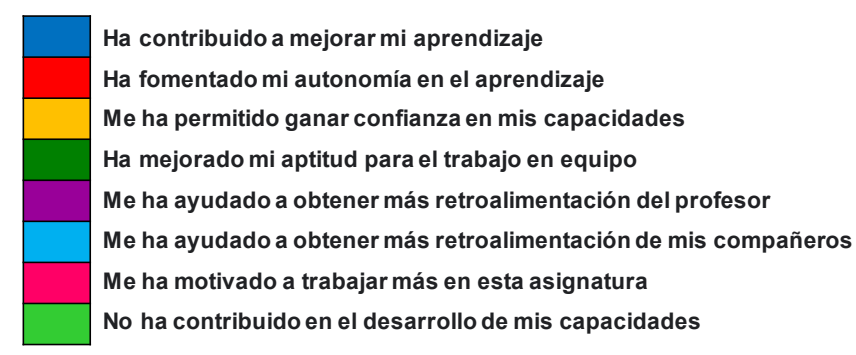

Figura 4. Análisis de capacidades en diferentes actividades de la asignatura.

- Sin embargo, ni los cuestionarios de coevaluación ni autoevaluación realizados al final de las tutorías ni las clases magistrales iniciales impartidas al principio de curso en la asignatura son bien acogidas por los estudiantes, habiendo una mayoría de estudiantes que consideran que no han contribuido al desarrollo de sus capacidades. En este último caso se debe a que se trata de un repaso de la teoría impartida en asignaturas de cursos previos necesaria para esta asignatura de LDI y los estudiantes consideran que no es necesario volver a repasar dicha teoría para desarrollar nuevas capacidades.

Por tanto, son las tutorías presenciales, las prácticas de laboratorio y los cuestionarios de autoevaluación en Moodle las actividades que, de acuerdo a la opinión de los estudiantes, han contribuido en mayor manera a mejorar su aprendizaje, mientras que la formación de grupos y el trabajo en laboratorio, son las que más han mejorado su aptitud ante el trabajo en equipo. Las actividades más motivantes han sido las tutorías de seguimiento y las prácticas de laboratorio. Los seminarios iniciales sobre repaso de contenidos y explicación de las plantas piloto parecen levantar opiniones opuestas ya que un 34\% considera que no ha aportado nada al desarrollo de sus capacidades mientras que el $30 \%$ considera que ha contribuido a mejorar su aprendizaje. Esto puede tener que ver con los conocimientos previos que tenga cada estudiante. De hecho, se ha comprobado en la encuesta sobre el perfil de los estudiantes, que tienen conocimientos dispares, puesto que muchos de ellos cursan esta asignatura sin haber superado otras recomendadas como Ingeniería de la Reacción Química u Operaciones de Separación.

Finalmente se preguntó a los estudiantes sobre la satisfacción global que tenían de esta asignatura. La mayor parte de los estudiantes, un $68 \%$, tienen una satisfacción media alta con respecto a la asignatura (valoración de 3 en una escala de 4) mientras que un $20 \%$ de los encuestados no se encuentran totalmente satisfechos con la asignatura (valoración de 2 en una escala de 4).

\subsection{Resultados de aprendizaje}

Por último, se presentan las calificaciones de varios aspectos de la asignatura de LDI, comparando los resultados de aprendizaje representados por la calificación numérica obtenida a final del presente curso $19 / 20$, donde se han introducido las nuevas metodologías docentes detalladas en este proyecto, respecto a los tres cursos anteriores en los que dichas metodologías no se emplearon. 
En primer lugar, e incidiendo de nuevo en las tutorías evaluativas de seguimiento, actividades de gran importancia en esta asignatura, se puede observar cómo la mayor parte de los estudiantes en el presente curso 19/20 tienen una calificación media de las 4 tutorías entre 7 y 7,9, valor superior al alcanzado en los cursos anteriores (Figura 5). Ello puede deberse al efecto de los cuestionarios realizados a los estudiantes previos a las tutorías que les ha puesto de manifiesto los puntos más importantes a tratar en cada tutoría y que ha servido para su autoevaluación antes de llevar a cabo la tutoría.

\section{Media tutorías}

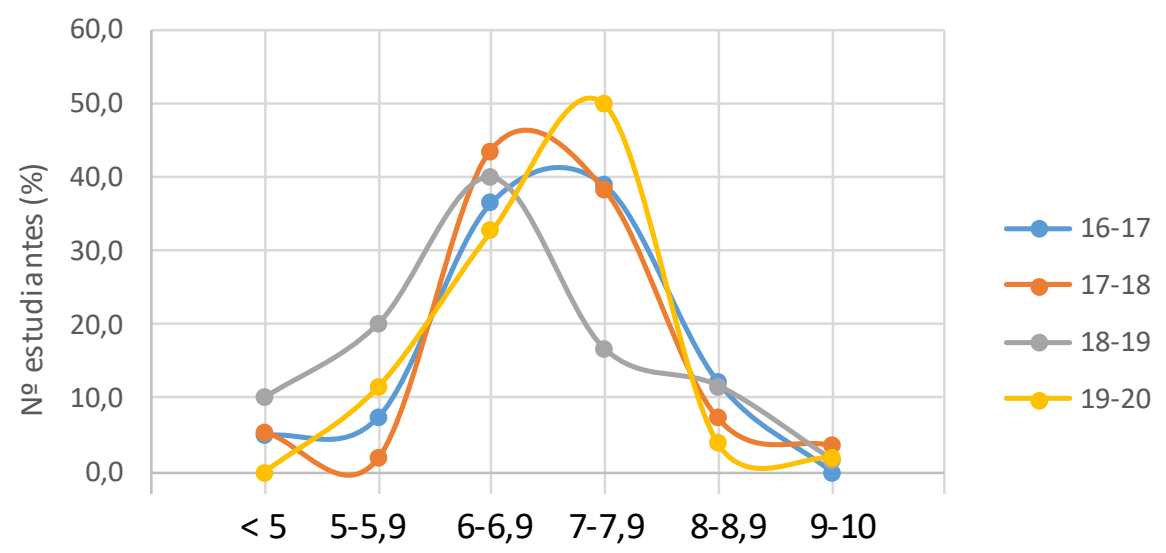

Figura 5. Calificaciones medias de las cuatro tutorías de seguimiento evaluativas realizadas durante el curso.

Por último, en la Figura 6, se observan las calificaciones finales obtenidas en la asignatura de LDI teniendo en cuenta todos los aspectos que en ella se contemplan: tutorías evaluativas, trabajo personal desarrollado en el laboratorio, informes escritos entregados durante el curso, tanto en grupo como individuales, y exámenes, tanto oral como escrito. Como puede observarse, también en este caso la calificación global lograda ha sido mayor, incrementándose sobre todo el número de alumnos que han conseguido una calificación final entre 8 y 8,9 .

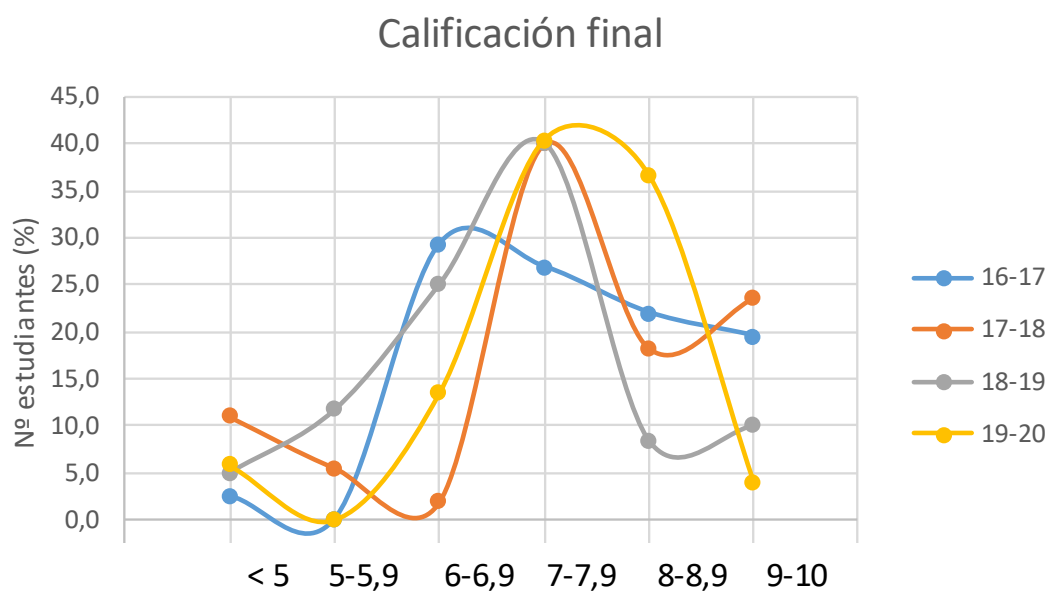

Figura 6. Calificaciones finales de la asignatura de LDI durante los últimos cursos impartidos. 
Por tanto, las metodologías activas introducidas en este curso parecen haber favorecido positivamente los resultados de aprendizaje y se considera conviene mantenerlas en cursos venideros con el fin de confirmar esta tendencia.

\section{Conclusiones}

El desarrollo del presente proyecto ha permitido introducir nuevas metodologías docentes en la asignatura Laboratorio de Desarrollo Industrial (LDI), de $4^{\circ}$ curso del Grado en Ingeniería Química de la Universidad Autónoma de Madrid. Las principales conclusiones que se pueden obtener del trabajo realizado son las siguientes:

- Conviene incidir en las ventajas del trabajo en equipo y de sus implicaciones en el desarrollo de proyectos para seguir potenciando los resultados, dado que las mejoras se han explicado este curso a partir de la motivación del grupo de trabajo y la confección de grupos homogéneos entre sí y compensados.

- Los cuestionarios previos a las tutorías han sido muy beneficiosos para los estudiantes porque les ha ayudado a discernir los aspectos más impotantes a tratar en cada tutoria y ha ayudado a plantear, por tanto, un mejor enfoque de la misma. Además, esta autoevaluación previa les ha permitido comprobar su nivel de preparación ante la inminente tutoría evaluativa. A los docentes les ha ayudado a conocer el nivel de preparación de los estudiantes antes de cada tutoría y, a través de estos cuestionarios, han podido reflejar aquellos temas que ellos consideraban más importantes para cada tutoría.

- Es necesario mejorar la percepción de los estudiantes sobre la comunicación y conocimientos que demuestran en las tutorías de seguimiento. Por ello, los docentes de la asignatura deberán incidir más en las rúbricas de evaluación que emplean, dedicando más tiempo a su explicación y comprobando que han sido entendidas por los estudiantes.

- Deben introducirse medidas de estímulo, en estudiantes y profesores, para fomentar el trabajo autónomo de los estudiantes y el segumineto online de los profesores, mediante el empleo de herramientas de gestión a distancia. De esta manera, se fomenta una mejor organización del trabajo por parte de alumnos y docentes y se garantiza una retroalimentación continua.

\section{Referencias}

BASILOTTA, V. y HERRADA, G. (2013). "Aprendizaje a través de proyectos colaborativos con TIC. Análisis de dos experiencias en el contexto educativo". Revista Electrónica de Tecnología Educativa, 44, $1-13$.

LERÍS LÓPEZ, D., LETOSA FLETA, J., USÓN SARDAÑA, A., ALLUEVA TORRES, P. y BUENO GARCÍA, C. (2017) "Trabajo en equipo y estilos de aprendizaje en la educación superior". Revista Complutense de Educación, 28(4), 1267-1284.

MARTÍ, J.A., HEYDRICH, M., ROJAS, M. y HERNÁNDEZ, A. (2010). “Aprendizaje basado en proyectos: una experiencia de innovación docente”. Revista Universidad EAFIT, 46(158), 11-21.

PARÍS MAÑAS, G., MAS TORELLÓ, O. y TORRELLES NADAL, C. (2016). "La evaluación de la competencia "trabajo en equipo" de los estudiantes universitarios". Revista d'Innovació Docent Universitària, 8, 86-97. 